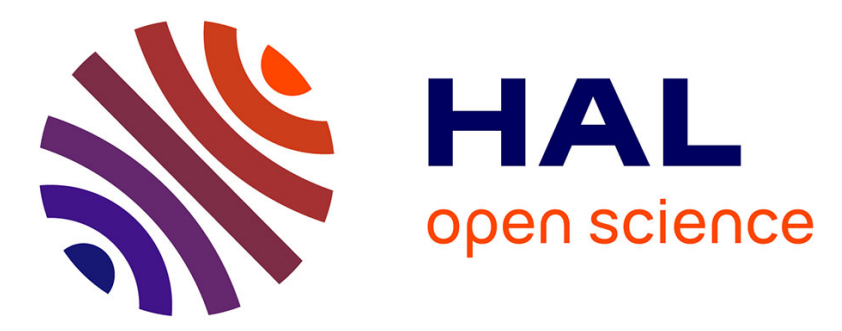

\title{
Electron energy distribution function in a low-power Hall thruster discharge and near-field plume
}

\author{
M. Tichý, A. Pétin, P. Kudrna, M. Horký, S. Mazouffre
}

\section{To cite this version:}

M. Tichý, A. Pétin, P. Kudrna, M. Horký, S. Mazouffre. Electron energy distribution function in a low-power Hall thruster discharge and near-field plume. Physics of Plasmas, 2018, 25 (6), pp.061205. 10.1063/1.5017578 . hal-02071675

\section{HAL Id: hal-02071675 \\ https://hal.science/hal-02071675}

Submitted on 27 Jan 2022

HAL is a multi-disciplinary open access archive for the deposit and dissemination of scientific research documents, whether they are published or not. The documents may come from teaching and research institutions in France or abroad, or from public or private research centers.
L'archive ouverte pluridisciplinaire HAL, est destinée au dépôt et à la diffusion de documents scientifiques de niveau recherche, publiés ou non, émanant des établissements d'enseignement et de recherche français ou étrangers, des laboratoires publics ou privés. 


\title{
Electron energy distribution function in a low-power Hall thruster discharge and near-field plume
}

\author{
M. Tichý ${ }^{1,2}$ A. Pétin, ${ }^{3}$ P. Kudrna, ${ }^{1}$ M. Horký, ${ }^{4}$ and S. Mazouffre ${ }^{3}$ \\ ${ }_{1}^{1}$ Faculty of Mathematics and Physics, Charles University, V Holešovičkách 2, 18000 Prague, Czech Republic \\ ${ }^{2}$ National Research Tomsk Polytechnic University, Lenin Avenue, 30, Tomsk 634050, Russia \\ ${ }^{3}$ Institut de Combustion, Aérothermique, Réactivité et Environnement, CNRS, 45071 Orléans cedex 2, France \\ ${ }^{4}$ Department of Space Physics, Institute of Atmospheric Physics CAS, Boční II 1401, 14131 Prague, \\ Czech Republic
}

(Received 28 November 2017; accepted 27 April 2018; published online 16 May 2018)

Electron temperature and plasma density, as well as the electron energy distribution function (EEDF), have been obtained inside and outside the dielectric channel of a $200 \mathrm{~W}$ permanent magnet Hall thruster. Measurements were carried out by means of a cylindrical Langmuir probe mounted onto a compact fast moving translation stage. The 3D particle-in cell numerical simulations complement experiments. The model accounts for the crossed electric and magnetic field configuration in a weakly collisional regime where only electrons are magnetized. Since only the electron dynamics is of interest in this study, an artificial mass of ions corresponding to $m_{i}=30000 m_{e}$ was used to ensure ions could be assumed at rest. The simulation domain is located at the thruster exit plane and does not include the cathode. The measured EEDF evidences a high-energy electron population that is superimposed onto the low energy bulk population outside the channel. Inside the channel, the EEDF is close to Maxwellian. Both the experimental and numerical EEDF depart from an equilibrium distribution at the channel exit plane, a region of high magnetic field. We therefore conclude that the fast electron group found in the experiment corresponds to the electrons emitted by the external cathode that reach the thruster discharge without experiencing collision events. Published by AIP Publishing. https://doi.org/10.1063/1.5017578

\section{INTRODUCTION}

Contrary to chemical propulsion, which is limited by the energy stored in the propellant, electric propulsion (EP) relies on external energy sources, currently the light emitted by the Sun, to accelerate the propellant to a very high velocity through a thermal expansion process or an electric or magnetic force; see Ref. 1 and references herein. As a consequence of the momentum conservation law, the high propellant ejection speed achieved by EP devices directly translates into a drastic reduction in the propellant mass required for a given mission or maneuver. Therefore, EP allows for the decrease of the cost of a space mission, the extension of space vehicle capabilities, and the realization of missions unfeasible with chemical engines. ${ }^{1,2}$ In addition, electric thrusters can be switched on and off on-demand, a relevant aspect for mission range, and they demonstrate a long operational life, which enables spacecraft to reach a high velocity. There is a vast set of EP technologies with various characteristics, some devices being well-advanced with an extensive flight heritage and others still in development stage. ${ }^{1}$ Currently, the most mature and most used technologies are the gridded ion engines and the Hall thrusters (HTs). ${ }^{3}$ Although the Hall thrusters deliver a specific impulse, i.e., an ion ejection speed, below that of ion engines, they produce a much higher thrust-to-power ratio $(\sim 60 \mathrm{mN} / \mathrm{kW})$ with an efficiency in excess of $50 \%$. The Hall thrusters with a power level in the $1-5 \mathrm{~kW}$ range are then well-suited for station keeping, attitude control, and orbit transfer of heavy communication satellites. The low-power HTs operating around $200 \mathrm{~W}$ represent an interesting option for the small satellites of mega-constellations such as the OneWeb constellation. ${ }^{4}$ Very high-power HTs are foreseen as main propulsion means for space tugs and cargos in the frame of several Moon and Mars exploration programs. ${ }^{5}$

The operation principle of a HT relies on a magnetic barrier and a low-pressure partially magnetized DC discharge generated between an anode placed in an annular dielectric chamber and an external hot cathode. ${ }^{1,3,6-8}$ The applied magnetic field is strong enough to make the electron Larmor radius much smaller than the chamber characteristic dimensions, but weak enough not to affect ion trajectories. The electric potential drop is localized in the final section of the chamber owing to the low axial mobility of electrons in the region of strong B field. The electric field governs the propellant atoms ionization and the ion acceleration, namely, the thrust and the specific impulse. The combination of the radial magnetic field with the axial electric field generates an $\mathrm{E} \times \mathrm{B}$ electron drift in the azimuthal direction, the so-called Hall current, which is responsible for the efficient ionization of the propellant atoms. Although the architecture and the basic principle of a HT are relatively simple, the physical mechanisms that govern the thrust generation and efficiency are not yet fully understood. ${ }^{1}$ This is mostly due to a lack of accurate insights into a diffusion of electrons through the magnetic barrier and into the interaction between plasma and surfaces. ${ }^{8-10}$ Despite enormous efforts on the fundamental and the experimental viewpoints, modelling and computer simulations are still not able to make reliable predictions whatever the approach, i.e., fluid, particle-in-cell, or hybrid. For the industry, a direct consequence of this lack of 
understanding is long development and qualification periods for new HT designs, which translates into high cost.

One way to move forward is to investigate the electron properties in the discharge and plume of the Hall thrusters. First, electrons play a key role in the ionization process. Second, the electron transport phenomena determine the electric field distribution. Therefore, electrons govern to a large extent the HT characteristics. Combining the experimental measurements of electron density and temperature with a numerical simulation appears to be a good strategy for improving the understanding of the Hall thruster physics. In this contribution, we present the measurements of floating and plasma potential, electron temperature, and density, and the Electron Energy Distribution Function (EEDF) carried out by a cylindrical Langmuir probe in the near-field plasma plume and inside the cavity of a low-power HT. Moreover, we complement the experimental outcomes by the numerical simulations based on a 3D Particle-In-Cell (PIC) model that accounts for crossed electric and magnetic field configuration in a weakly collisional regime.

\section{LOW-POWER HALL THRUSTER}

In this work, the EEDFs have been measured in the discharge and in the plasma plume near-field of the ISCT200 Hall thruster. ISCT is an acronym for ICARE Small Customizable Thruster. The ISCT200 is a versatile $200 \mathrm{~W}$ class Hall thruster using permanent magnets for generating the magnetic field instead of helical magnetizing coils. ${ }^{11-13}$ The ISCT200 delivers $12 \mathrm{mN}$ of thrust and $1100 \mathrm{~s}$ of specific impulse at $200 \mathrm{~W}$ with an anode efficiency of $35 \%$. The main characteristics of the ISCT200 Hall thruster are the following. The annular channel external diameter is about $50 \mathrm{~mm}$. The channel geometry is in the so-called $2 \mathrm{~S}_{0}$ configuration, which means the channel width-to-mean diameter ratio is twice the standard one, where the standard ratio is defined as that of the well-known Russian SPT100 thruster. As demonstrated in the preceding studies with the $200 \mathrm{~W}$ and $100 \mathrm{~W}$ Hall thrusters, a broad channel improves the performance level of the low-power HTs in terms of thrust, mass utilization, ion production cost, ion velocity, thermal load, and operation envelope. ${ }^{11}$ The inner and outer channel walls are made of ${\mathrm{BN}-\mathrm{SiO}_{2}}_{2}$ ceramic. A set of SmCo permanent magnets combined with a soft iron magnetic circuit generate the magnetic field topology. The magnetic field exhibits a bell-shape distribution along the channel centerline with the largest strength at the channel exit plane. The propellant gas is injected at the back of the dielectric channel using a metal injector placed behind the ceramic through a circular aperture. The anode is a $5 \mathrm{~mm}$ in width and $1 \mathrm{~mm}$ in thickness steel ring placed at the back of the channel against the internal surface of the outer dielectric wall. Copper heat pipes evacuate the heat towards a large radiator installed at the back of the thruster body. The steady-state temperature of the thruster inner pole is about $125^{\circ} \mathrm{C}$ at $220 \mathrm{~W}$ input power, while the magnet temperature is about $185^{\circ} \mathrm{C}$, well below the SmCo Curie point.

A heated hollow cathode with a disk-shaped $\mathrm{LaB}_{6}$ emitter was used to generate the electron current needed for discharge balance and ion beam neutralization. The cathode is placed outside the channel underneath the thruster. It is located $10 \mathrm{~cm}$ away from the symmetry axis in the radial direction and $2 \mathrm{~cm}$ away from the channel exit plane in the axial direction. The magnetic field strength at the cathode orifice is around $2 \mathrm{G}$, slightly above the Earth's magnetic field. Assuming the electron temperature $\mathrm{T}_{\mathrm{e}}=1 \mathrm{eV}$ at the cathode outlet, the Larmor radius amounts to about $20 \mathrm{~mm}$, so electrons are not strongly magnetized, yet they attach to B field lines that surround the cathode and move towards the anode. The cathode is electrically connected to the thruster anode. The cathode and the thruster body are floating but unbound. High-purity Xenon (Xe) was used as the propellant gas for both the thruster discharge and the cathode discharge. The cathode has been operated with a constant Xe mass flow rate of $0.2 \mathrm{mg} / \mathrm{s}$. Typically, about $150 \mathrm{~W}$ electrical power is applied to the cathode to sufficiently heat-up the emitting element. A photograph of the ISCT200 is shown in Fig. 1.

All experiments have been performed in the cryogenically pumped NExET (New Experiments on Electric Thrusters) vacuum chamber. The NExET is based on a $1.8 \mathrm{~m}$ in length and $0.8 \mathrm{~m}$ in diameter stainless steel tank. Primary pumping is ensured by a dry pump that evacuates $400 \mathrm{~m}^{3} / \mathrm{h}$. A $3501 / \mathrm{s}$ turbomolecular pump is capable of further evacuating the chamber down to $10^{-6}$ mbar- $\mathrm{N}_{2}$, by absorbing light gases such as $\mathrm{N}_{2}, \mathrm{O}_{2}, \mathrm{H}_{2} \mathrm{O}$, and $\mathrm{H}_{2}$. The cryogenic pump absorbs the gases such as $\mathrm{Xe}$ and $\mathrm{Kr}$. The pumping speed is around $8000 \mathrm{l} / \mathrm{s}$ when the $0.5 \mathrm{~m}^{2}$ cold plate is sustained at $35 \mathrm{~K}$. The overall pump stack warrants a background pressure as low as $2 \times 10^{-5}$ mbar-Xe during the operation of a $200 \mathrm{~W}$ input power plasma source. The heavy particle momentum exchange mean free path is, in this backpressure condition, on the order of the chamber length, which guarantees a negligible impact of the residual gas on the source operation and measurement outcomes. A large water-cooled screen covered with graphite tiles is mounted at the back of the chamber. It absorbs a part of the ion beam energy, therefore reducing the thermal load onto the cryogenic surface.

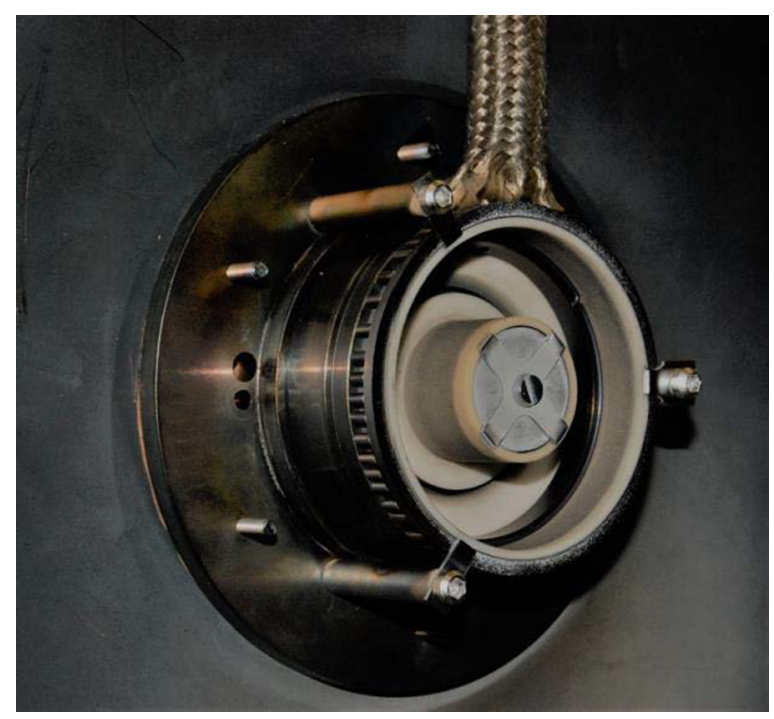

FIG. 1. Picture of the $200 \mathrm{~W}$-class permanent magnet ISCT200 Hall thruster. 
The chamber is equipped with several observation windows, access ports, and vacuum feed-through connectors for power lines, gas lines, and diagnostic tools. A large front door enables easy access to the interior of the vessel.

\section{MEASURING INSTRUMENT}

\section{A. Langmuir probe}

The properties of electrons in the plasma of the Hall thrusters can be effectively studied by means of electric probes. In addition to being a relatively simple method, probes allow to locally determine the quantities such as the electron density and temperature along with the EEDF. There are several types of electric probes for plasma discharge investigation, among others the Langmuir probe, ${ }^{14}$ the double probe ${ }^{15}$ the triple probe, ${ }^{16}$ and the emissive probe. ${ }^{17}$ Since the power deposition on the probe is high in our case, especially when measuring inside the thruster channel, the probes are installed on a fast moving translation stage, as described in Ref. 18. In order to follow high-frequency plasma instabilities, the probe circuits have to minimize the influence of stray capacitances; see, e.g., Ref. 19.

The electron energy distribution function (EEDF), or the electron energy probability function (EEPF), is certainly the most interesting electron-related quantity, since it describes both the electron density and the electron mean energy also in the case when the EEDF deviates from Maxwellian. In the vicinity of the thruster exit plane, the authors report in Ref. 14 the non-Maxwellian EEDF with a distinct second maximum at comparatively higher electron energies. The resulting "doubly humped" EEDF is explained in Ref. 14 as a sum of the two functions- the magnetized beam electron distribution function (Maxwellian function with a drift) and the Maxwellian plasma electron distribution function. The qualitative agreement with this form of the EEDF close to the thruster is also reported in the recent Monte Carlo model. ${ }^{20}$ In the following text, we use both the notions of the EEPF$\mathrm{f}(\mathrm{E})$ and $\operatorname{EEDF}-\mathrm{F}(\mathrm{E})$; their relation is $f(E)=F(E) E^{-1 / 2}$, where $\mathrm{E}$ denotes the energy. ${ }^{21}$

Our Langmuir probe architecture was similar to the one described in Ref. 22. The Langmuir probe was made of a tungsten wire $0.2 \mathrm{~mm}$ in diameter. The non-collecting part of the wire was insulated from the plasma by a $100 \mathrm{~mm}$ long and $2 \mathrm{~mm}$ in diameter alumina tube. The length of the collecting part was $1 \mathrm{~mm}$. There were three reasons for making the probe so short, namely, the spatial resolution, the high plasma density, and the expected plasma anisotropy. With a short probe, the shape of the sheath-the area collecting the charged particles for the probe-resembles a sphere and effectively averages the current from all the directions. Since the probe became red hot (i.e., its surface was at approximately $800^{\circ} \mathrm{C}$ ) when measuring inside the thruster channel, we confirmed with the alumina manufacturer that the material keeps its insulating properties at least up to $1500^{\circ} \mathrm{C}$.

In this work, the Langmuir probe was mounted onto a linear piezo drive that was placed outside the thruster plume. The used linear drive was a PILine Linear ${ }^{\circledR}$ Motor Stage M664K018 with a speed up to $35 \mathrm{~cm} / \mathrm{s}$; a more detailed description can be found in Ref. 18. The linear drive was placed under a graphite cover in order to protect it against direct ion bombardment and to prevent the motor to be extensively heated. The motor moves the probe to the measurement position and then the acquisition of the I-V characteristic starts. After the measurement, the probe is moved back to its rest position. In order to minimize the wear of the probe due to probe material sputtering, the measurement time/sweep rate was kept as short as possible, typically 10-30 s. For collecting the probe data, we have used the Advanced Langmuir Pobe (ALP) system from Impedans, Ltd.

This system uses a fast A-D converter capable to process up to 80 megasamples per second with 16-bit resolution. We used a $0.5 \mathrm{~V}$ voltage step and, depending on the EEDF energy range, we acquired between 113 and 287 points (voltage steps) per probe characteristic. Each point of the probe characteristic was averaged over several millions of individual measurements for at least $50 \mathrm{~ms}$. The data acquisition duration for each point is well above the breathing mode oscillations time period, i.e., around $50 \mu$ s. Therefore, we performed genuine time-averaged measurements. The plasma potential was estimated from the abscissa of the maximum of the first derivative of the probe characteristic.

\section{B. Specifics}

It is worthwhile to note that Langmuir probe measurements in the magnetized plasmas are difficult to perform, and in general, there is anisotropy between the electron temperatures parallel and perpendicular to the magnetic field lines. In the method used here for the determination of the EEDF, the two electron temperatures are effectively averaged to generate a single distribution that is a function of the electron energy only, and which thus only has a single (effective) temperature. This is consistent with the isotropic assumption often used for the electrons in many fluid based models. $^{23}$

Another effect that complicates the EEDF measurements are the plasma instabilities that are characteristic of the Hall thrusters. ${ }^{24}$ These fluctuations are of stochastic nature, and their frequency spectrum ranges from several $\mathrm{kHz}$ (breathing mode oscillations, see, e.g., Ref. 24) up to several MHz (transit time oscillations, see, e.g., Ref. 25). Since we decided to perform the time-averaged probe measurements, we had to be sure not to misinterpret the "humped" structure of the measured EEDF as that one might have resulted from insufficient probe frequency compensation. ${ }^{26}$ Fortunately, the perturbations induced by electrostatic probe in the discharge of the Hall thrusters have already been studied by comparing probe and laser measurements. ${ }^{27}$ It was found that the discharge suddenly changes to a more stable behavior when the probe was positioned approximately half a channel width upstream and downstream of the thruster exit plane. For the ISCT200 thruster-that is very similar to the one studied in Ref. 27this corresponds to approximately $\pm 5 \mathrm{~mm}$ with respect to the channel exit plane. In addition, the effect of oscillations on the probe characteristic decreases sharply when the electron energy increases. ${ }^{26,28}$ Consequently, we are confident that our time-averaged probe measurements, especially in view on the high-energy electron beams appearing on the EEDF, are not 
significantly influenced by the plasma instabilities. The lowenergy part of the EEDF, however, especially that near the plasma potential (zero energy), might be affected by the fluctuations of the plasma potential in the near-field and inside the thruster, where the fluctuation amplitude is large. The plasma potential fluctuations "smear"/degrade the lowenergy part of the EEDF that is reflected on the lower value of the electron density estimated from the probe electron current at the plasma potential as well as from the integral of the EEDF. That is why we also have estimated the positive ion density using the well-known Allen-Boyd-Reynolds (ABR) theory by Allen et al. ${ }^{29}$ That can be performed inside the thruster where the measured EEDF is close to Maxwellian, since that is assumed by the ABR theory. The plasma density estimated from the positive ion current is not much affected by the plasma potential fluctuations since its dependence on the probe voltage is weak. In the far-field plume, the oscillation amplitude is small enough to assume no significant influence on the electron density estimation.

It has been shown in Ref. 30 by LIF that the timeaveraged electric field distribution on the channel axis has a double-peaked shape. The profile was then confirmed by the time-resolved measurements. We are then confident that the observed double-peaked EEDFs are not the result of the timeaveraged measurements. A further rationale for that is given in Fig. 6(b) that shows the dependence of the energy of the "EEDF hump" (the high-energy peak) with the distance. This trace, namely, follows (within the experimental uncertainties) the change in the "electron accelerating voltage," i.e., the difference between the plasma and the cathode potential, with the distance, see Fig. 6(b). The plasma velocity does not change so dramatically within the studied range of distances as to generate the measured energy shift of the "hump."

The secondary electron emission (SEE) from the probe surface can apparently reduce the probe electron current, thus leading to lower values of the electron density. This effect has been studied experimentally in Refs. 31 and 32 and found significant at higher pressures for Knudsen numbers $K_{i, e}=\lambda_{i, e} / r_{p} \lesssim 1\left(\lambda_{i, e}\right.$ denotes the mean free path of ions and electrons, respectively, and $r_{p}$ the probe radius). In our experimental conditions, $K_{i}$ is typically greater than 1000 and $K_{e}$ greater than 60000 . Hence, we assume the effect of SEE on the electron density estimation is negligible.

We could not exactly assess the probable effect of sputtering of the probe material and of the ceramic probe holder by high energy xenon ions. As mentioned in Sec. III A, in order to minimize the effects due to the probe material sputtering, the presence of the probe in the thruster channel was kept as short as possible.

\section{PARTICLE-IN-CELL MODEL}

To simulate electron dynamics in the thruster, we used the self-consistent Particle In Cell (PIC) code DiP3D developed at the University of Oslo. This PIC code works with the realistic dimensional parameters at the input which are then normalized for computation. Although dimensionless quantities are used during computations, at the end the results are scaled back to SI units. Because the code is electrostatic, it does not count for the perturbations in magnetic field; thus, it cannot simulate the electromagnetic phenomena. DiP3D code uses a Cartesian three dimensional grid where all three components of the particle position and velocity are calculated. It also allows to set external stationary electric and magnetic fields in the arbitrary directions and accounts for collisions between the charged particles and neutrals using the null collision algorithm. ${ }^{33,34}$ We have chosen the 3D code instead of a 2D code because of more realistic resolution of electron motion. Particle trajectories are calculated with leap-frog scheme combined with Boris algorithm. ${ }^{35}$ Poisson equation $\Delta \varphi=-\frac{\rho}{\varepsilon_{0}}$ for electric potential is solved by Multigrid method. Electric field on the grid is therefore calculated from the electric potential using finite difference method of the equation $\boldsymbol{E}=-\nabla \varphi$. In the simulations, we use first order weighting for both charge density to grid and electric field from the grid to simulate particles. The first order weighting is sufficient to maintain a numerical stability in such simulations where the electron gyroradius is well resolved on the grid. ${ }^{36}$ The code as well as its tests with regard to numerical noise is described in more detail in the previous publications. ${ }^{37-39}$

For our simulations, we used Cartesian coordinate system and periodic boundary conditions, which means that the values of electric potential generated by particles were the same on the opposite sides and edges of simulation box. We simulated the volume with physical dimension $3 \times 3 \times 3 \mathrm{~cm}^{3}$ located in the center of exit plane of thruster, schematically depicted in Fig. 2. At that location, the electric and magnetic fields can be assumed as perpendicular; thus, we set external magnetic field in the $\mathrm{x}$-direction and electric field in the $\mathrm{y}$-direction resulting in plasma drift in the negative z-direction. Since we were interested in electron dynamics, we used artificial mass of ions corresponding to $M_{i}=30000 m_{e}$ which ensured that ions could be assumed as immobile. Neutral atoms are not simulated as particles but the collision of

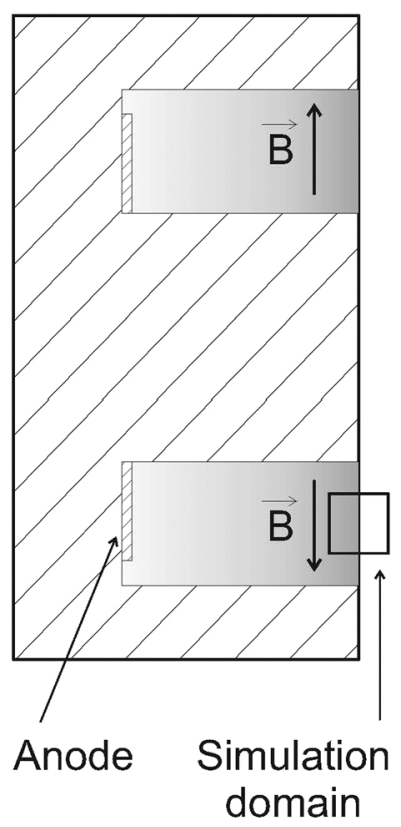

FIG. 2. Location of the simulation domain. 
TABLE I. Parameters of simulation.

\begin{tabular}{lcc}
\hline \hline Parameter & Value & Units \\
\hline Box size & $0.03 \times 0.03 \times 0.03$ & $\mathrm{~m}$ \\
Grid size & $64 \times 64 \times 64$ & $\ldots$ \\
Delta t (time step) & $5.11 \times 10^{-11}$ & $\mathrm{~s}$ \\
B0 & 0.00015 & $\mathrm{~T}$ \\
E0 & $140,220,350$ & $\mathrm{~V} / \mathrm{cm}$ \\
$n_{i}, n_{e}$ (ion, electron densities) & $4.000000 \times 10^{13}$ & $1 / \mathrm{m}^{3}$ \\
$N_{i}, N_{e}$ (number of macroparticles) & 5000000 & $\ldots$ \\
$M_{i} / m_{e}$ & 30000 & $\ldots$ \\
$T_{e}$ (electron temperature) & 10 & $\mathrm{eV}$ \\
$\lambda_{D}$ (Debye length) & $1.661846 \times 10^{-3}$ & $\mathrm{~m}$ \\
Number of timesteps & $1.0 \times 10^{5}$ & $\ldots$ \\
\hline \hline
\end{tabular}

electrons with neutrals is provided by Monte Carlo algorithm with a constant collision frequency $\nu=2.6 \times 10^{7} \mathrm{~s}^{-1}$, which corresponds to the electron gyrofrequency. In the Hall thrusters, the collision frequency for electron cross-field transport is indeed close to the gyrofrequency, see, e.g., Ref. 40. Since the collision frequency varies significantly along the channel, see Fig. 5 in Ref. 40, we also performed calculations with two other values; the results are reported in the following chapter. As the first approach and for the simplicity, we do not assume the collisions between ions and neutral background as well as other types (ionization, etc.) of electronneutral collisions. We used the time step $\Delta t=5.11 \times 10^{-11} \mathrm{~s}$, which was sufficient to resolve the cyclotron motion, as well as the plasma frequency of electrons; the total length of simulation was 100000 time steps corresponding to approximately 135 electron gyroperiods. Grid spacing used in the simulations is sufficient to resolve both the electron Debye length and electron gyroradius, which are the conditions required for numerical stability of the simulation. The number of simulated particles (electrons and ions) is constant throughout the simulation. Both electrons and ion are generated with the three dimensional Maxwellian distributions.

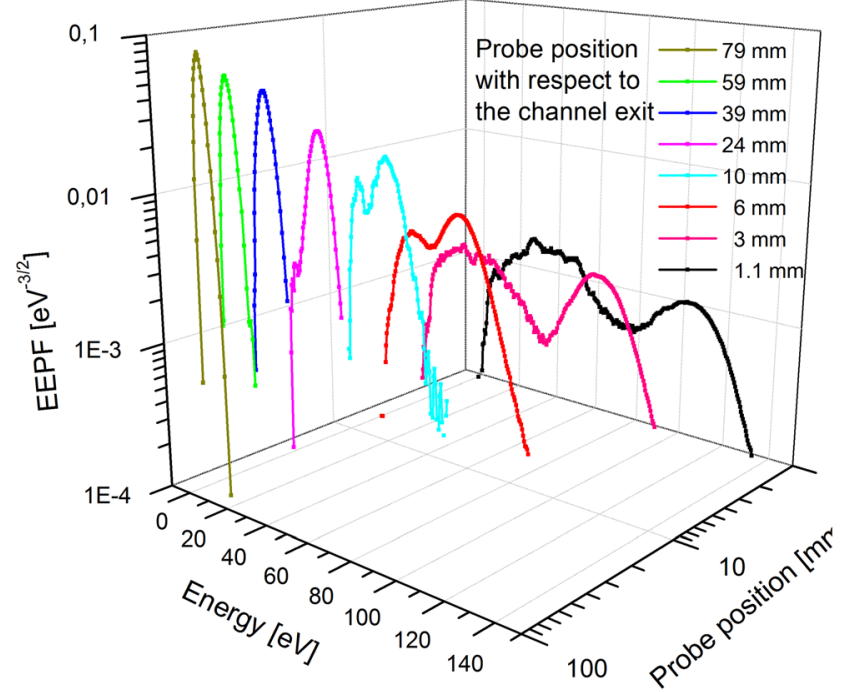

(a)
The true plasma parameters at the thruster exit plane have been scaled down as a result of compromise between the accuracy, numerical stability, and speed of the simulations. However, the plasma to gyrofrequency ratio as well as the drift $v_{d}$ to thermal velocity ratio is conserved, which means their value corresponds to the real situation. The simulation parameters are given in Table I. While the parameters are chosen predominantly according to numerical stability and performance, their characteristic ratios (e.g., temperature, frequencies, and velocities) can be related to realistic values, such as those found at the outlet of a low-power thruster.

\section{RESULTS AND DISCUSSION}

The ISTC200 thruster was operating at a discharge voltage $200 \mathrm{~V}$ and $1 \mathrm{mg} / \mathrm{s}$ Xe throughput. The cathode potential relative to ground was $-20 \mathrm{~V}$. The measurements were performed on the axis of the thruster channel at probe positions ranging from the far field region $(+79 \mathrm{~mm})$ down to the channel interior $(-7.9 \mathrm{~mm})$. Notice the $x=0 \mathrm{~mm}$ position refers to the thruster channel exit plane and negative values indicate locations inside the channel.

The EEPF has been measured at 15 discrete positions ( 8 upstream and 7 downstream of the channel exit). Figure 3 shows the measured EEPF as a function of the distance from the thruster exit plane; the left panel shows the measurements downstream of the channel exit plane and the right panel the measurements inside the thruster channel. The group of fast electrons is being formed close to the thruster channel exit plane. At about $1 \mathrm{~mm}$ downstream of the thruster exit plane, the EEPF has a "double humped" form. The reason for such a behavior has been explained in several publications, see, e.g., Ref. 14. The high-energy group corresponds to the electrons originating directly from the cathode and experiencing none or just a few collision events. We believe that our PIC model further supports this statement concluded in Ref. 14. Such electrons follow the magnetic

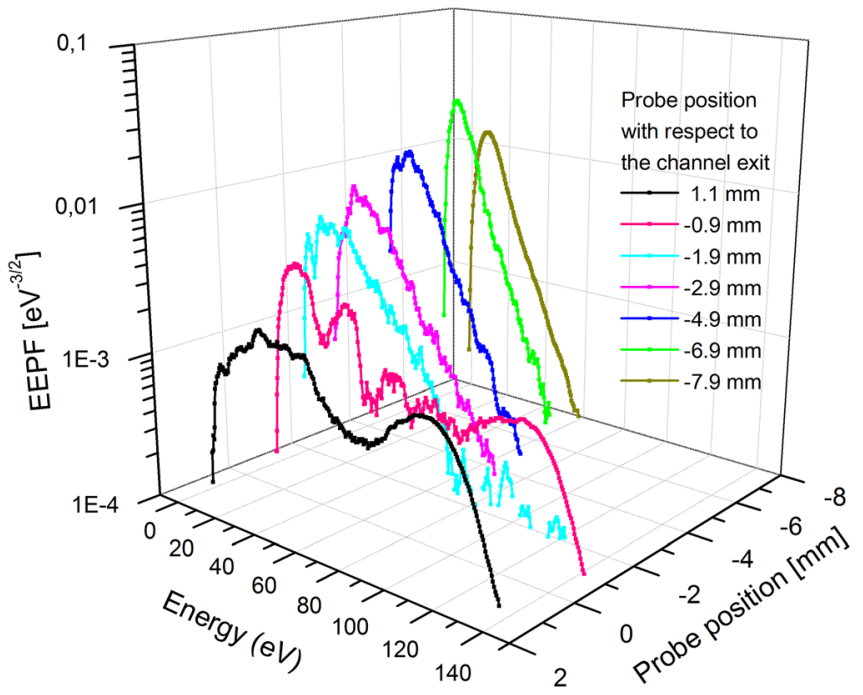

(b)

FIG. 3. The measured EEPFs as a function of the probe position with respect to the channel exit. The EEPFs are normalized to 1. Left panel: downstream positions. Right panel: positions inside the channel. The curve for position $1.1 \mathrm{~mm}$ is displayed for the reference in both panels. 


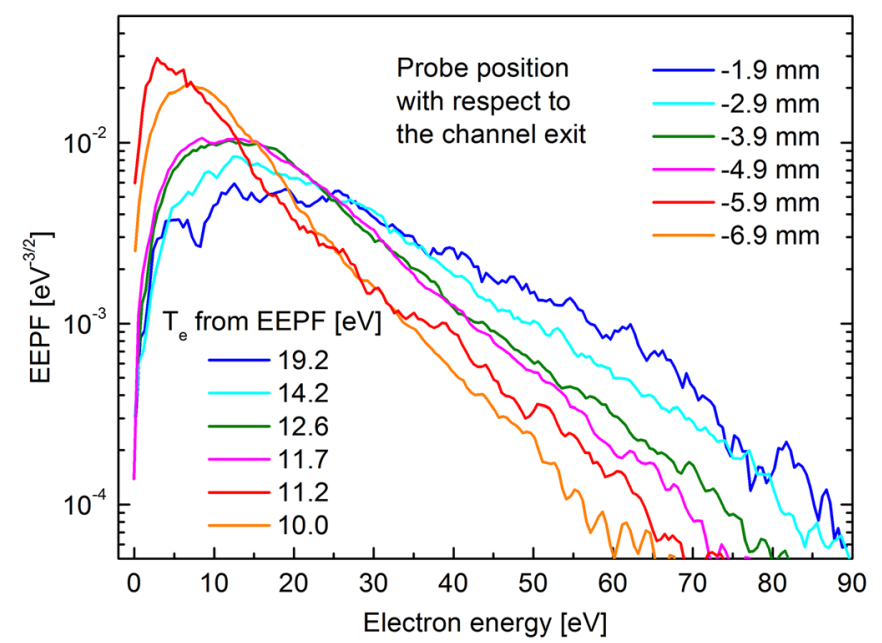

FIG. 4. The EEPF in absolute scale (normalized to 1) inside the thruster channel. The linearity of the EEPF body in semilogarithmic scale indicates that the EEDF is close to Maxwellian; the respective electron temperatures are also given.

field line that intercepts the cathode, and they experience very few collisions with atoms, hence their high energy. These electrons become involved in a strong azimuthal $\mathrm{E} \times \mathrm{B}$ drift and their energy peaks at about $\mathrm{E}=120 \mathrm{eV}$ forming a hump on the tail of the EEDF. At almost the same position, i.e., at the channel outlet vicinity, there is a group of low energy electrons that peaks at around $40 \mathrm{eV}$. It is assumed that it consists of electrons that are produced inside the thruster channel and subsequently accelerated by the electric field at the thruster exit, of which the amplitude is around $110 \mathrm{~V} / \mathrm{mm}$, see Fig. 6(a). Inside the thruster, the "double-humped" form of the EEPF changes to Maxwellian when the electron temperature decreases in the upstream direction. On the curve recorded at $\mathrm{x}=-0.9 \mathrm{~mm}$, see Fig. 3, the local maxima observed at lower energies are probably caused by high level of noise on the probe characteristic at this position. However, we believe that the local maximum on the EEPF at around $120 \mathrm{~V}$ is real since the effect of oscillations on the probe characteristic decreases sharply with an increasing electron energy. ${ }^{26}$

Further downstream of the thruster exit plane, the fast electron group gradually merges into the main body of the EEDF as shown in Fig. 3. Starting from approximately $20 \mathrm{~mm}$ downstream of the thruster exit, the second electron group ceases to be measurable, and only the decreasing width and the increasing sharpness of the form of the EEDF indicates the decrease in the mean electron energy in the downstream direction.

When the EEDF becomes Maxwellian at larger downstream distances, the electron cooling can be described by the laws of thermodynamics. It is itself an interesting phenomenon, since the electron cooling in expanding the magnetized plasma appears to be collisionless. The electron cooling in a magnetically expanding plasma has been described in several current and earlier publications, see, e.g., Ref. 41.

The EEPFs obtained at six discrete positions inside the thruster channel are displayed in Fig. 4. The EEPFs are displayed in absolute scale as they were obtained by double numerical differentiation of the measured probe characteristics with respect to the probe voltage, and the integral of each EEPF over all the indicated electron energies was normalized to one. It is seen that from approximately $-2 \mathrm{~mm}$ upstream of the thruster exit plane, the EEPF is close to Maxwellian with decreasing electron temperature [see also Fig. 7(b)]. That reflects in our opinion the influence of the electron-electron collisions that play a critical role in the relaxation time of the EEPF towards a Maxwellian, see, e.g., Ref. 42. The contour plot in Fig. 5 shows clearly the development of the EEDF over the whole measured range of distances.

Figure 6(a) shows the floating $\left(\mathrm{V}_{\mathrm{fl}}\right)$ and plasma $\left(\mathrm{V}_{\mathrm{pl}}\right)$ potential as a function of the distance. Figure 6(b) shows the

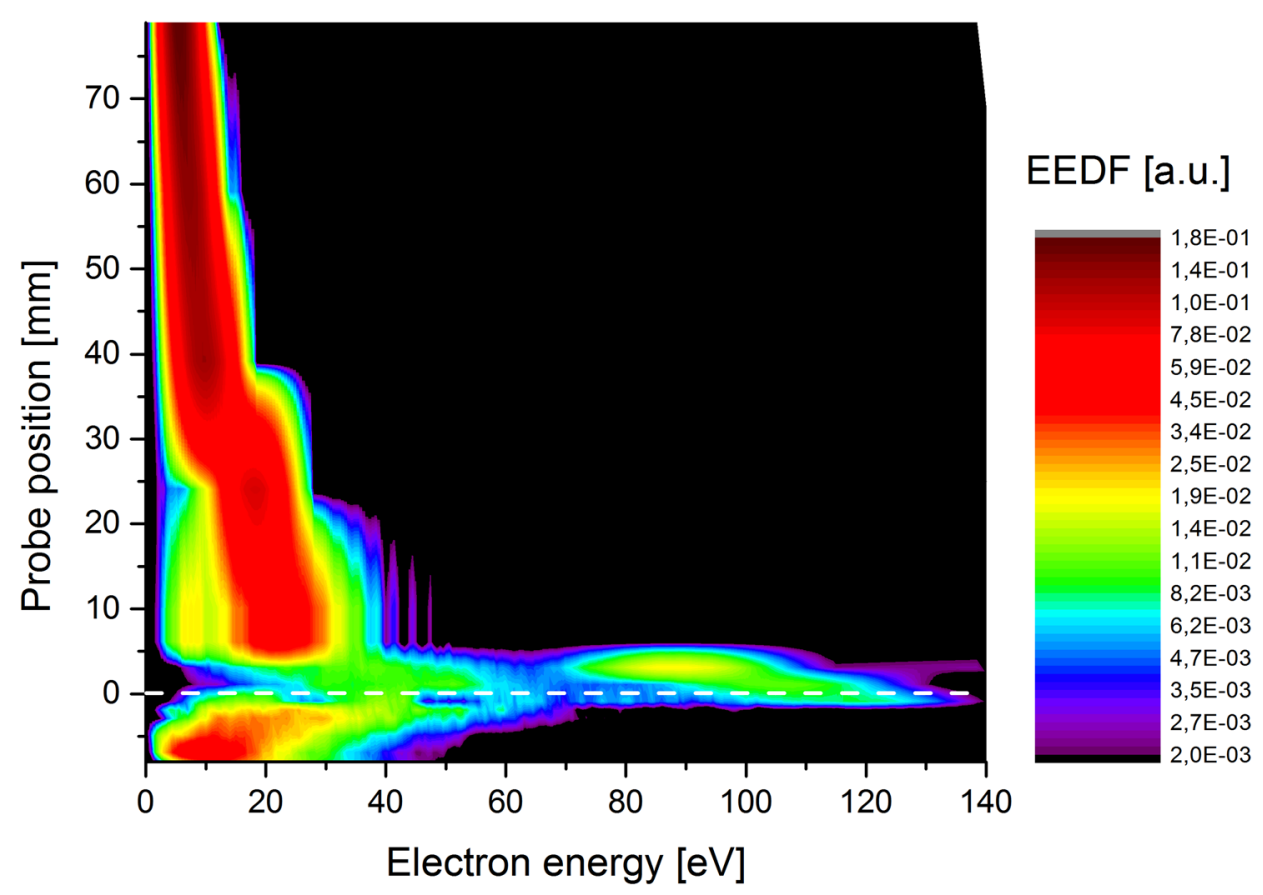

FIG. 5. Measured EEDF as a function of the distance from the thruster exit plane (dashed line at $x=0 \mathrm{~mm}$ ). 

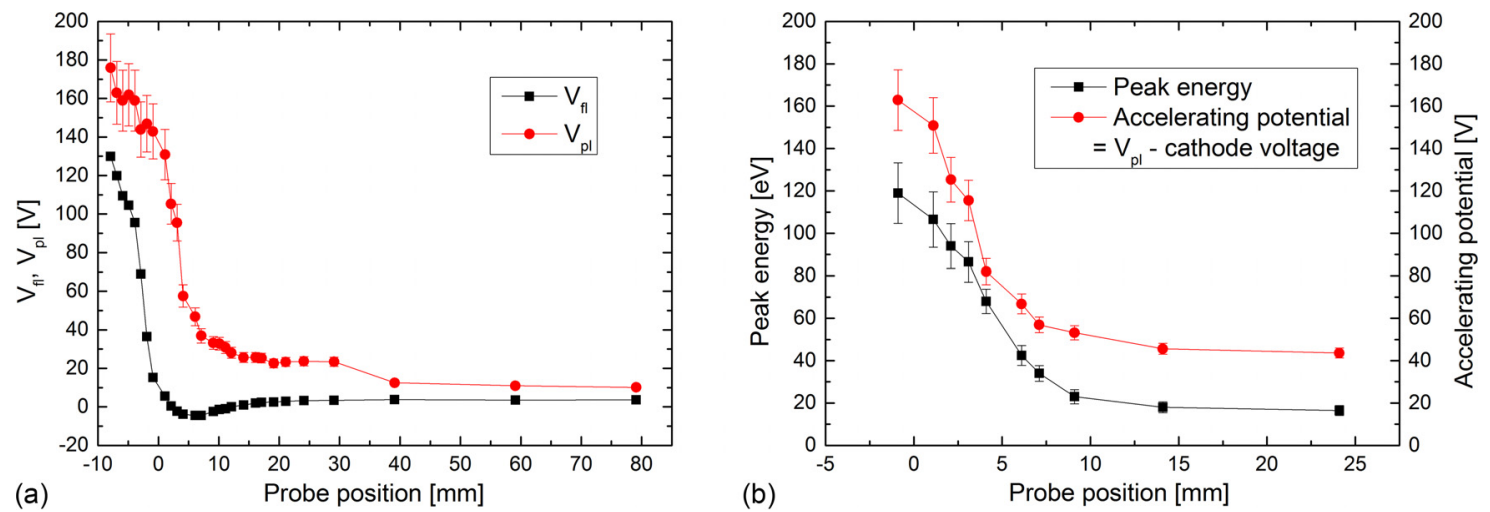

FIG. 6. (a) The floating and plasma potential as a function of the probe position. (b) Dependence of the high-energy peak of the EEDF on the probe position. For comparison is also depicted the accelerating potential.

variations of the energy of the high-energy peak of the EEDF with the probe position. We assume that these energetic electrons come from the cathode and are accelerated by the voltage difference between the cathode set at $-20 \mathrm{~V}$ and the plasma potential; Fig. 6(b) well documents that.

Figures 7(a) and 7(b) show the electron density and electron temperature against the distance, respectively. The estimation of the charged particles density in a fluctuating plasma is burden with error as we explained in Sec. III B. In order to gather information about the measurement error, we have estimated the electron as well as the positive ion density. The points displayed in Fig. 7(a) were obtained as the average values estimated from the electron as well as from the ion part of the probe characteristic using the theory. ${ }^{29}$ The error bars represent the spread between the apparent density values estimated by the different methods. While at larger distances where the oscillation amplitude is comparatively small, the measurement error amounts to acceptable $\pm 20 \%-30 \%$, and at the thruster exit plane, the error is bigger, approximately $\pm 40 \%$. The plasma potential has been determined from the abscissa of the maximum of the first derivative. Figure 7(b) shows the electron temperature $T_{e}$. The electron temperature was estimated by "classical" method from the slope of the semilogarithmic plot of the electron current probe characteristic. In other words, the $\mathrm{T}_{\mathrm{e}}$ in Fig. 7(b) characterizes the low-energy Maxwellian part of the EEDF that carries the majority of electrons. When getting closer to the thruster, the number of electrons inside the highenergy tail of the EEPF increases that is seen, apart from the high-energy peaks on the EEPF, also on the difference between the floating and the plasma potential in Fig. 6(a); the fast electrons shift the floating potential to more negative values. ${ }^{43}$ From Fig. 7(b), it is clear that the electron energy increases in the upstream direction, i.e., towards the thruster interior. However, it does not reach its maximum at the thruster exit plane, but at about $-3 \mathrm{~mm}$ inside the thruster. This position approximately coincides with the maximum of the magnetic field strength. ${ }^{44}$ Roughly at the same position, i.e., at around $-3 \mathrm{~mm}$ inside the thruster channel, we observe the maximum of the electron density, see Fig. 7(a). The second local maximum of the electron density occurs at approximately $16 \mathrm{~mm}$ downstream of the thruster exit plane and it probably indicates the place where the electrons emitted from the hot cathode join the Xe ion beam. Alternatively, it may indicate the focusing feature of the ISCT200 thruster. ${ }^{44}$

The results from the simulations are shown in Fig. 8. The initial velocity distribution $\mathrm{F}\left(u_{z} / v_{d}, u_{y} / v_{d}\right)$ of electrons in a plane perpendicular to the external magnetic field is expected to be a usual bi-Maxwellian type normalized to the drift velocity $v_{d}$. The electron energy distributions $\mathrm{F}(\mathrm{E})$ taken from the half time of simulation are depicted in semilogarithmic scale in Fig. 8. They were calculated for the three values of the electric field $\mathrm{E}$ at the channel exit plane: $140 \mathrm{~V} / \mathrm{cm}$, $220 \mathrm{~V} / \mathrm{cm}$, and $350 \mathrm{~V} / \mathrm{cm}$. With a $200 \mathrm{~V}$ applied discharge
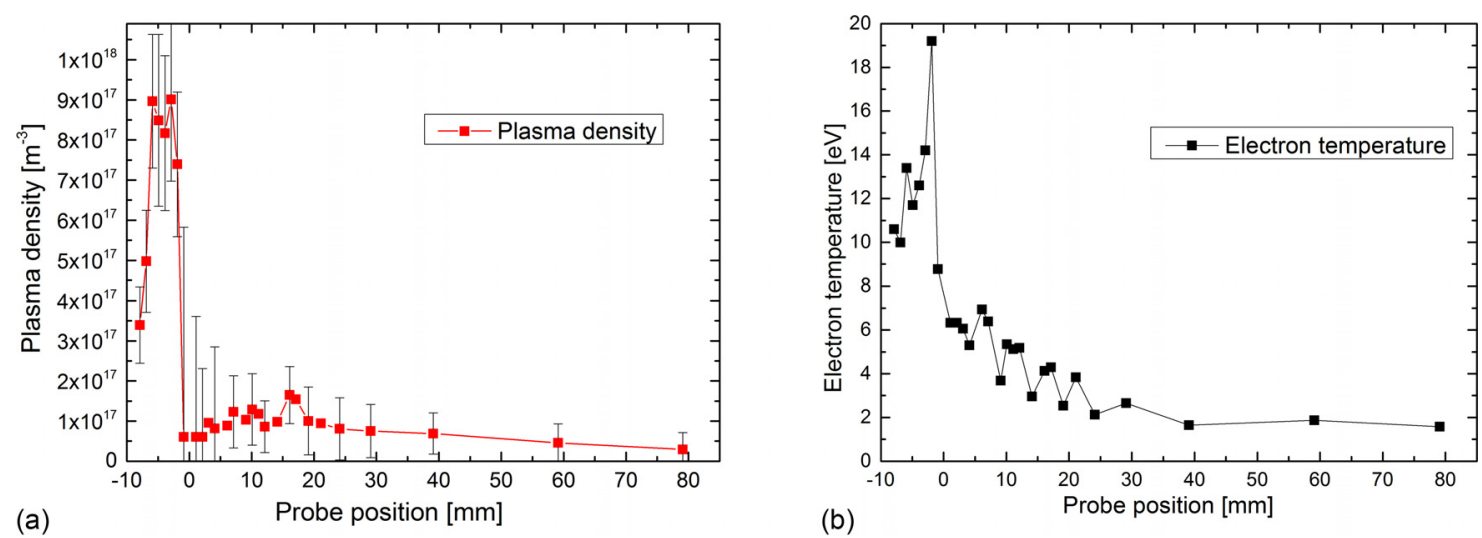

FIG. 7. The electron/positive ion density (a) and the electron temperature (b) as a function of the probe position. 


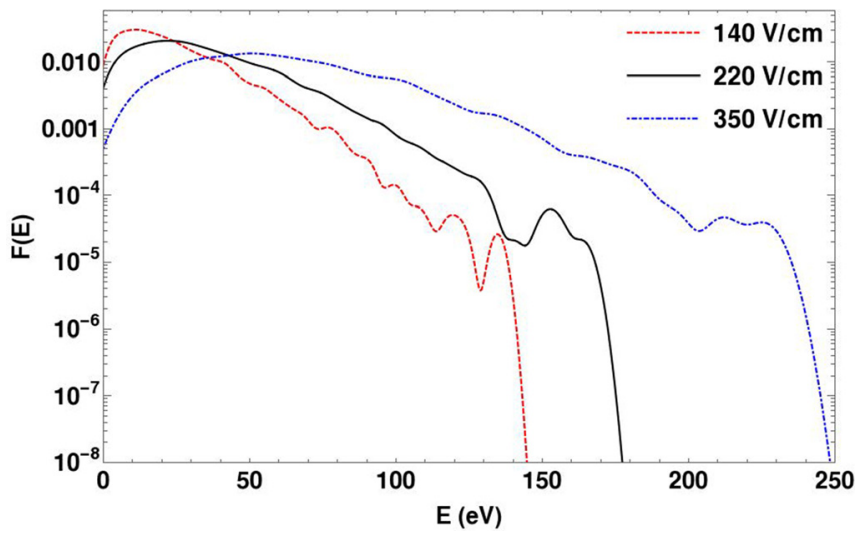

FIG. 8. The electron energy distributions from the simulation in semilogarithmic scale at three different electric fields at the thruster exit plane.

voltage, we measured a $\sim 220 \mathrm{~V} / \mathrm{cm}$ steady-state E field magnitude at the channel exit plane; i.e., the $\mathrm{E} \approx 220 \mathrm{~V} / \mathrm{cm}$ corresponds to the thruster operating conditions during our Langmuir probe measurements.

The form of the calculated EEDF at all three E-field values is close to Maxwellian with the electron temperature rising with the raising electric field: $16.4 \mathrm{eV}, 21.2 \mathrm{eV}$, and $41.0 \mathrm{eV}$, respectively. Simultaneously, each EEDF shows a peak at the $\sim 130 \mathrm{eV}, \sim 150 \mathrm{eV}$, and $\sim 230 \mathrm{eV}$ electron energy, respectively. The measured EEPF at the electric field magnitude at the channel exit plane $\sim 220 \mathrm{~V} / \mathrm{cm}$ and at the distance, $-0.9 \mathrm{~mm}$ from the thruster exit plane shows a second peak at $\sim 120 \mathrm{eV}$. That is in fair agreement with the calculation considering that the local electric field and, consequently, the energy of the second group of electrons in the EEPF can change strongly with the position close to the thruster exit plane. The amplitudes of the high-energy peaks on the computed EEDFs are smaller with respect to those found on the experimental EEDF; however, their amplitude is well above the noise level that occurs in our PIC simulations. ${ }^{36}$ Hence, the fast electron groups on the EEDFs estimated experimentally correspond to the electrons emitted by the cathode and accelerated by the difference between the cathode and the plasma potential almost without collisions with the ions in the plasma plume. The PIC model yielded also the spectrum of potential fluctuations. The computed spectrum showed a wave activity at lower frequencies that might correspond to the breathing mode. However, low frequencies were not well resolved in our simulation.

As reported in Ref. 40, classical collision theory predicts electron-neutral collision frequency values across the magnetic field lines that are much lower than those required for reproducing plasma properties and thruster performances. Besides, the electron-neutral collision frequency is a critical aspect of the mechanism we propose for the origin of the high-energy population in the $\mathrm{F}(\mathrm{E})$. In order to find out how the collision frequency influences the simulated $F(E)$, we performed the calculations with a collision frequency by one order of magnitude higher $\left(\nu=2.6 \times 10^{8} \mathrm{~s}^{-1}\right)$ and by one order of magnitude lower $\left(\nu=2.6 \times 10^{6} \mathrm{~s}^{-1}\right)$ than that used in Fig. $8\left(\nu=2.6 \times 10^{7} \mathrm{~s}^{-1}\right)$. For the simulation, we chose the magnitude of the electric field that corresponded to our

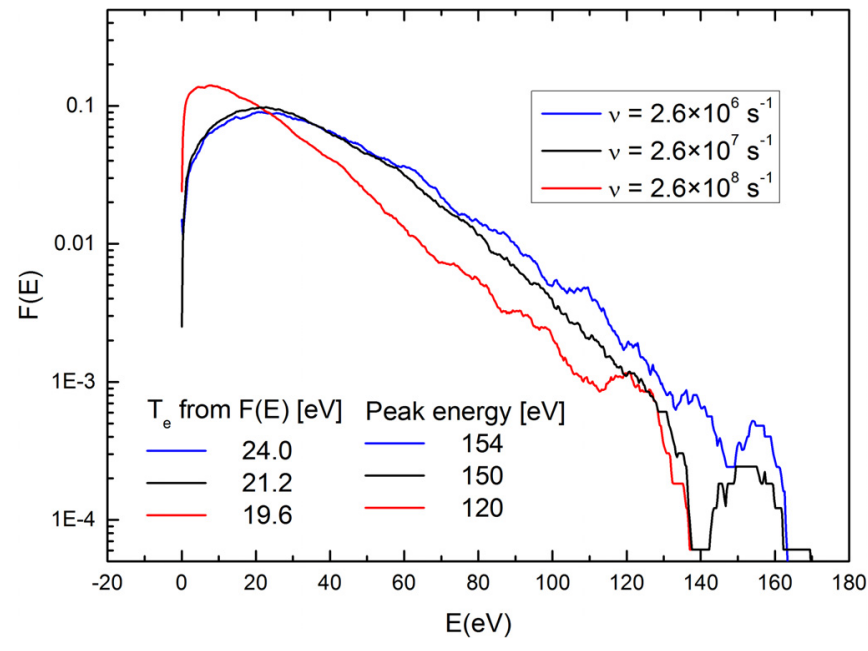

FIG. 9. The electron energy distributions from the simulation in semilogarithmic scale at three different electron-neutral collision frequencies at the thruster exit plane.

experiment, $\mathrm{E}=220 \mathrm{~V} / \mathrm{cm}$. The results are shown in Fig. 9 along with the original curve from Fig. 8. The feature to note is the approximate electron temperature that decreases with increasing the collision frequency; the collisions cool down the electrons. Furthermore, the form of the $F(E)$ is closer to Maxwellian with higher collision frequency. That is also understandable, since the neutral particles have Maxwellian distribution. Finally, the high-energy peak on the $F(E)$ at higher collision frequency appears at lower energy and at $\nu=2.6 \times 10^{8} \mathrm{~s}^{-1}$ its position corresponds to that found experimentally: $120 \mathrm{eV}$. That result corresponds qualitatively to the conclusion reported in Ref. 40 where it was found that the e-n collision frequency sharply rises at the boundary between the thruster exit and the near-field plume.

\section{CONCLUSIONS}

As it has been observed in Ref. 20, a better understanding of the Hall thruster physics requires a detailed study of the electron energy distribution function (EEDF) outside as well as inside of the thruster channel. This study hence aimed at complementing the experimental studies that investigated the far-field Hall thruster plasma $^{22,23,25,45}$ by an experimental study of the Hall thruster plasma in the nearfield and inside the thruster channel.

We are aware that the EEPF might be anisotropic especially inside the thruster channel, see, e.g., Refs. 23, 46, and 47. In the method used here for the determination of the EEPF, the anisotropy is effectively averaged by using the probe that has similar length as the diameter and its collecting area resembles therefore that of a spherical probe. We are also confident that our EEPF time-averaged measurements are not significantly influenced by the plasma instabilities as described in Ref. 26; inside the thruster and in the near-field plume, the presence of the probe reduces the oscillations. ${ }^{27}$ In addition, the high-energy beams appeared on the EEPFs at rather high energies and the effect of oscillations on the probe characteristic decreases sharply with increasing the electron energy. ${ }^{26,28}$ Further downstream, the oscillations 
do not have sufficient amplitude to influence the EEPF as it was proved by the previous measurements. ${ }^{18,22,48}$

Experiments confirm the idea that the EEPF near the thruster exit plane is composed of two electron groups. The first group is extracted from the external hollow cathode by the electric field having a component along the magnetic field near the cathode region. ${ }^{14,49}$ When arriving at the channel exit, these electrons are deflected by the strong radial magnetic field near the thruster exit, become involved in an $\mathrm{E} \times \mathrm{B}$ drift motion, and contribute to the ionization and sustaining of the thruster discharge. This magnetized beam of electrons has in our ISCT200 thruster an energy around $120 \mathrm{eV}$ when the applied potential is $200 \mathrm{~V}$. The second group consists of slower electrons produced by ionization inside the thruster channel and accelerated by the electric field at the channel exit. The EEPF's measured downstream of the thruster outlet gives a logical picture about how the magnetized beam of electrons merges into the EEPF body. At larger distances, the EEPF becomes Maxwellian and it is characterized by a decreasing electron temperature in the downstream direction.

Although our study shows the results similar to those presented in Ref. 14, a direct comparison between the two works is, unfortunately, very difficult.

The geometry of the SPT-50 thruster as described in Ref. 50 is different from that of ISCT200 as well as wall material, anode geometry, and magnetic field topology and strength. Besides, there is no information in Ref. 50 concerning the development of the plasma potential along the axis of the thruster channel from which one could deduce the voltage drop that accelerates the magnetized electron beam coming from the cathode. Our results, however, agree qualitatively with those presented in Ref. 14 in the following: (i) EEDF measurements reveal a high-energy electron beam of which the energy fairly corresponds to the potential difference between the hollow cathode and the thruster exit plane and (ii) the electron beam disappears when moving deeper inside the channel.

As we mentioned in Sec. III B, our Langmuir probe influences the plasma of the thruster when positioned close to the thruster exit plane or inside the thruster. Consequently, the EEPFs measured inside the thruster, see Fig. 4, do not characterize exactly the "natural mode" of the thruster discharge. On the other hand, the qualitative agreement with the outcomes of works published in Ref. 14 suggests that the thruster operation was not substantially degraded by the insertion of our measuring probe. Clearly, our experimental data are of relevance for comparison with the numerical simulations of an HT discharge.

The PIC model describes the thruster plasma only at the exit of the channel. Even if it was necessary to scale down the real plasma parameters in order to achieve a reasonable computational time, the three calculated EEDFs also show a group of fast electrons; its amplitude is, however, lower than that obtained from experiment. The simulation performed at three different e-n collision frequencies confirmed the expected behavior of the EEDF with the e-n collision frequency. We therefore conclude that the fast electron group found in the experiment corresponds to the electrons emitted by the external cathode that reach the thruster discharge without experiencing the collision events.

\section{ACKNOWLEDGMENTS}

M.T. and P.K. acknowledge the partial financial support from the Czech Science Foundation, Grant No. 15-00863S. M.H. acknowledges the support from the Premium Academiae Award from the Czech Academy of Sciences. Numerical simulations were carried out using DiP3D code at the UiO Abel supercomputer through project Notur/NorStore NN9299K.

${ }^{1}$ S. Mazouffre, "Electric propulsion for satellites and spacecraft: Established technologies and novel approaches," Plasma Sources Sci. Technol. 25, 033002 (2016).

${ }^{2}$ R. H. Frisbee, "Advanced space propulsion for the 21st century," J. Propul. Power 19, 1129-1154 (2003).

${ }^{3}$ D. M. Goebel and I. Katz, Fundamentals of Electric Propulsion (Wiley, Hoboken, NJ, 2008).

${ }^{4}$ I. Levchenko, K. Bazaka, Y. Ding, Y. Raitses, S. Mazouffre, T. Henning, P. J. Klar, S. Shinohara, J. Schein, L. Garrigues, M. Kim, D. Lev, F. Taccogna, R. W. Boswell, C. Charles, H. Koizumi, S. Yan, C. Scharlemann, M. Keidar, and S. Xu, "Space micropropulsion systems for Cubesats and small satellites: From proximate targets to furthermost frontiers," Appl. Phys. Rev. 5, 011104 (2018).

${ }^{5}$ S. J. Hall, B. A. Jorns, A. D. Gallimore, H. Kamhawi, T. W. Haag, J. A. Mackey, J. H. Gilland, P. Peterson, and M. J. Baird, "High-power performance of a 100-kW class nested Hall thruster," in Proceedings of the 35th International Electric Propulsion Conference, Atlanta, Georgia, 2017 IEPC Paper No. 2017-228, and references herein.

${ }^{6}$ J.-P. Boeuf, "Tutorial: Physics and modeling of Hall thrusters," J. Appl. Phys. 121, 011101 (2017).

${ }^{7}$ V. Zhurin, H. Kaufmann, and R. Robinson, "Physics of closed drift thrusters," Plasma Sources Sci. Technol. 8, R1-R20 (1999).

${ }^{8}$ N. Gascon, M. Dudeck, and S. Barral, "Wall material effects in stationary plasma thrusters. I. Parametric studies of an SPT-100," Phys. Plasmas 10, 4123-4136 (2003).

${ }^{9}$ J. C. Adam, A. Héron, and G. Laval, "Study of stationary plasma thrusters using two-dimensional fully kinetic simulations," Phys. Plasmas 11, 295 (2004).

${ }^{10}$ A. Héron and J. C. Adam, "Anomalous conductivity in Hall thrusters: Effects of the non-linear coupling of the electron cyclotron drift instability with secondary electron emission of the walls," Phys. Plasmas 20, 082313 (2013).

${ }^{11}$ S. Mazouffre, G. Bourgeois, K. Dannenmayer, and A. Lejeune, "Ionization and acceleration processes in a small, variable channel width, permanent magnet Hall thruster," J. Phys. D: Appl. Phys. 45, 185203 (2012).

${ }^{12}$ L. Grimaud and S. Mazouffre, "Conducting wall Hall thrusters in magnetic shielding and standard configurations," J. Appl. Phys. 122, 033305 (2017).

${ }^{13}$ L. Grimaud, S. Mazouffre, and C. Boniface, "Performance comparison between standard and magnetically shielded $200 \mathrm{~W}$ Hall thrusters with $\mathrm{BN}-\mathrm{SiO} 2$ and graphite channel walls," in Proceedings of the 35th International Electric Propulsion Conference, Atlanta, Georgia, 2017, Paper No. IEPC-2017-172.

${ }^{14}$ V. Yu. Fedotov, A. A. Ivanov, G. Guerrini, A. N. Vesselovzorov, and M. Bacal, "On the electron energy distribution function in a Hall-type thruster," Phys. Plasmas 6, 4360 (1999).

${ }^{15}$ B. Beal, L. Johnson, D. Brown, J. Blakely, and D. Bromaghim, "Improved analysis techniques for cylindrical and spherical double probes," Rev. Sci. Instrum. 83, 073506 (2012).

${ }^{16}$ B. E. Beal, A. D. Gallimore, J. M. Haas, and W. A. Hargus, "Plasma properties in the plume of a Hall thruster cluster," J. Propul. Power 20, 985 (2004).

${ }^{17}$ A. W. Smith and M. A. Cappelli, "Time and space-correlated plasma potential measurements in the near field of a coaxial Hall plasma discharge," Phys. Plasmas 16, 073504 (2009).

${ }^{18}$ K. Dannenmayer and S. Mazouffre, "Compact high-speed reciprocating probe system for measurements in a Hall thruster," Rev. Sci. Instrum. 83, 123503 (2012)

${ }^{19}$ R. B. Lobbia and A. D. Gallimore, "High-speed dual Langmuir probe," Rev. Sci. Instrum. 81, 073503 (2010). 
${ }^{20}$ F. Taccogna, R. Schneider, S. Longo, and M. Capitelli, "Kinetic simulations of a plasma thruster," Plasma Sources Sci. Technol. 17, 024003 (2008).

${ }^{21}$ V. A. Godyak, R. B. Piejak, and B. M. Alexandrovich, "Measurements of electron energy distribution in low pressure RF discharges," Plasma Sources Sci. Technol. 1, 36 (1992).

${ }^{22}$ K. Dannenmayer, P. Kudrna, M. Tichy, and S. Mazouffre, "Time-resolved measurement of plasma parameters in the far-field plume of a low-power Hall effect thruster," Plasma Sources Sci. Technol. 21, 055020 (2012).

${ }^{23}$ T. Lafleur, F. Cannat, J. Jarrige, P. Q. Elias, and D. Packan, "Electron dynamics and ion acceleration in expanding-plasma thrusters," Plasma Sources Sci. Technol. 24, 065013 (2015).

${ }^{24}$ J. P. Boeuf and L. Garrigues, "Low frequency oscillations in a stationary plasma thruster,” J. Appl. Phys. 84, 3541 (1998).

${ }^{25}$ J. Vaudolon and S. Mazouffre, "Observation of high-frequency ion instabilities in a cross-field plasma," Plasma Sources Sci. Technol. 24, 032003 (2015).

${ }^{26}$ S. Klagge, "Space- and direction-resolved Langmuir probe diagnostic in RF planar discharges," Plasma Chem. Plasma Process. 12, 103 (1992).

${ }^{27}$ L. Grimaud, A. Pétin, J. Vaudolon, and S. Mazouffre, "Perturbations induced by electrostatic probe in the discharge of Hall thrusters," Rev. Sci. Instrum. 87, 043506 (2016).

${ }^{28}$ S. Klagge and M. Maas, "The influence of the fluctuation amplitude on the probe characteristic," Beitr. Plasmaphys. 23, 355 (1983).

${ }^{29}$ J. E. Allen, R. L. F. Boyd, and P. Reynolds, "The collection of positive ions by a probe immersed in a plasma," Proc. Phys. Soc., London, Sect. B 70, 297 (1957).

${ }^{30}$ J. Vaudolon, B. Khiar, and S. Mazouffre, "Time evolution of the electric field in a Hall thruster," Plasma Sources Sci. Technol. 23, 022002 (2014).

${ }^{31}$ S. Klagge and M. Tichý, "A contribution to the assessment of the influence of collisions on the measurements with Langmuir probes in the thick sheath working regime," Czech. J. Phys. B 35, 988 (1985).

${ }^{32}$ E. Passoth, P. Kudrna, C. Csambal, J. F. Behnke, M. Tichý, and V. Helbig, "An experimental study of plasma density determination by a cylindrical Langmuir probe at different pressures and magnetic fields in a cylindrical magnetron discharge in heavy rare gases," J. Phys. D: Appl. Phys. 30, 1763 (1997)

${ }^{33}$ V. Vahedi and M. Surendra, "A Monte Carlo collision model for the particle-in-cell method: Applications to argon and oxygen discharges," Comput. Phys. Commun. 87, 179 (1995).

${ }^{34}$ H. R. Skullerud, "The stochastic computer simulation of ion motion in a gas subjected to a constant electric field,” J. Phys. D: Appl. Phys. 1, 1567 (1968).

${ }^{35}$ J. P. Boris, "Relativistic plasma simulation-optimization of a hybrid code," in Proceedings of 4th Conference on Numerical Simulation of Plasmas (Naval Res. Lab.) (1970), pp. 3-67.
${ }^{36}$ M. Horký, W. J. Miloch, and V. A. Delong, "Numerical heating of electrons in particle-in-cell simulations of fully magnetized plasmas," Phys. Rev. E 95, 043302 (2017).

${ }^{37}$ D. Block and W. J. Miloch, "Charging of multiple grains in subsonic and supersonic plasma flows," Plasma Phys. Controlled Fusion 57, 014019 (2015).

${ }^{38}$ W. J. Miloch, "Simulations of several finite-sized objects in plasma," Procedia Comput. Sci. 51, 1282-1291 (2015).

${ }^{39}$ W. J. Miloch, M. Kroll, and D. Block, "Charging and dynamics of a dust grain in the wakefield of other grain," Phys. Plasmas 17, 103703 (2010).

${ }^{40}$ A. L. Ortega and I. G. Mikellides, "The importance of the cathode plume and its interactions with the ion beam in numerical simulations of Hall thrusters," Phys. Plasmas 23, 043515 (2016).

${ }^{41}$ M. Martinez-Sanchez, J. Navarro-Cavallé, and E. Ahedo, "Electron cooling and finite potential drop in a magnetized plasma expansion," Phys. Plasmas 22, 053501 (2015).

${ }^{42}$ I. P. Shkarofsky, T. W. Johnston, and M. P. Bachynski, The Particle Kinetics of Plasmas, 1st ed. (Addison-Wesley Pub. Co., 1966).

${ }^{43}$ M. Cercek, G. Filipic, T. Gyergyek, and J. Kovacic, "Floating potentials in two-electron temperature plasma with two species of positive ions: Kinetic model and PIC simulation," Contrib. Plasma Phys. 50, 909 (2010).

${ }^{44}$ J. Vaudolon, "Electric field determination and magnetic topology optimization in Hall thrusters," Ph.D. thesis, Université d'Orléans, 2015.

${ }^{45}$ R. W. Boswell, K. Takahashi, C. Charles, and I. D. Kaganovich, "Nonlocal electron energy probability function in a plasma expanding along a magnetic nozzle," Front. Phys. 3, 14 (2015).

${ }^{46}$ S. W. Qing, H. Li, X. G. Wang, M. J. Song, and D. R. Yu, "Effect of anisotropic non-Maxwellian electron distribution function on plasma-wall interaction in Hall thrusters," Europhys. Lett. 100, 35002 (2012).

${ }^{47}$ F. Zhang, Y. Ding, H. Li, X. Wu, and D. Yu, "Effect of anisotropy of electron velocity distribution function on dynamic characteristics of sheath in Hall thrusters," Phys. Plasmas 18, 103512 (2011).

${ }^{48}$ K. Dannenmayer, P. Kudrna, M. Tichy, and S. Mazouffre, "Time-resolved measurements of plasma properties using electrostatic probes in the crossfield discharge of a Hall effect thruster," Contrib. Plasma Phys. 53, 63 (2013).

${ }^{49}$ S. Tsikata, N. Lemoine, V. Pisarev, and D. Grésillon, "Dispersion relations of electron density fluctuations in a Hall thruster plasma, observed by collective light scattering," Phys. Plasmas 16, 033506 (2009).

${ }^{50}$ G. Guerrini, C. Michaut, M. Bacal, A. N. Vesselovzorov, and A. A. Pogorelov, "An intense Hall-type ion source for satellite propulsion," Rev. Sci. Instrum. 69, 804 (1998). 